\title{
The principal parametric resonance of coupled van der pol oscillators under feedback control
}

\author{
Xinye $\mathrm{Li}^{\mathrm{a}, *}$, Huabiao Zhang ${ }^{\mathrm{b}}$ and Lijuan $\mathrm{He}^{\mathrm{c}}$ \\ ${ }^{a}$ School of Mechanical Engineering, Hebei University of Technology, Tianjin, P.R. China \\ ${ }^{\mathrm{b}}$ School of Astronautics, Harbin Institute of Technology, Harbin, P.R. China \\ ${ }^{\mathrm{c}}$ School of Mechanical Engineering, Tianjin University of Science and Technology, Tianjin, P.R. China
}

Received 9 August 2010

Revised 15 December 2010

\begin{abstract}
The principal parametric resonance of two van der Pol oscillators under coupled position and velocity feedback control with time delay is investigated analytically and numerically on the assumption that only one of the two oscillators is parametrically excited and the feedback control is linear. The slow-flow equations are obtained by the averaging method and simplified by truncating the first term of Taylor expansions for those terms with time delay. It is found that nontrivial solutions corresponding to periodic motions exist only for one oscillator if no feedback control is applied although the two oscillators are nonlinearly coupled. Based on Levenberg-Marquardt method, the effects of excitation and control parameters on the amplitude of periodic solutions of the system are graphically given. It can be seen that both of the two oscillators can be excited in periodic vibration with proper feedback. However, the amplitudes of the periodic vibrations are independent of the sign of feedback gains. In addition, the influence of time delay on the response of the system is periodic. In terms of numerical simulations, it is shown that both of the two oscillators can also have quasi-periodic motions, periodic motions about a new equilibrium position and other complex motions such as relaxation oscillation when feedback control is considered.
\end{abstract}

Keywords: Principal parametric resonance, coupled van der Pol oscillators, feedback control, time delay, bifurcation

\section{Introduction}

The behavior of a pair of coupled limit cycle oscillators or a system with two degrees of freedom displays a significantly wider range of phenomenon than a single limit cycle oscillator [1,2]. In recent two decades, research has shifted towards the study of the behavior of a system with two degrees of freedom including coupled limit cycle oscillators under parametrical excitation [3]. Maccari extended the asymptotic perturbation method to the case of two nonlinearly coupled and parametrically excited van der Pol oscillators without [4] and with [5] internal resonance respectively. Bakri et al. [6] explored a system with two degrees of freedom under parametric excitation by using both the harmonic balance method and the normal form method of averaging. Fatimah et al. [7] studied the bifurcation of a system which consists of an oscillator, coupled with a parametrically excited subsystem. Han Wei et al. [8] examined the effects of feedback parameters on the response of a nonlinear controlled system with 1:3 internal resonance and principal parametric resonance by the method of multiple scales. Ye Min et al. [9] dealt with the bifurcation of a laminated composite plate with 1:1 internal resonance and principal parametric resonance by the method of multiple scales and numerical method. Zhang Wei et al. [10] investigated the periodic and chaotic

\footnotetext{
*Corresponding author: Xinye Li, 13072210228 (mobile phone); E-mail: xinyeli@ hebut.edu.cn.
} 
oscillation of a parametrically excited viscoelastic moving belt with 1:3 internal resonance by the method of multiple scales and numerical method. Bi [11] discussed the dynamical behavior of two parametrically excited van der Pol oscillators with 1:1 internal resonance based on the averaging method. Recently, the effect of feedback control with time delay on dynamics of such complex systems has become a new interest of investigators [12]. ELBassiouny [13] studied the fundamental resonance and subharmonic resonance of order one-half of a harmonically forced oscillation under state feedback control with a time delay by using the multiple scale perturbation technique. Li Jun et al. [14] investigated the high-amplitude response suppression of the primary resonance of a nonlinear plant under cubic velocity feedback by means of the multiple scales method.

In this paper, the dynamical behavior of the system described by Eq. (1), with 1:1 internal resonance and only one oscillator parametrically excited, is presented.

$$
\begin{aligned}
& \ddot{y}_{1}+\omega_{1}^{2} y_{1}-2 \varepsilon f y_{2} \cos \Omega t-\varepsilon\left(1-y_{1}^{2}-\alpha_{2} y_{2}^{2}\right) \dot{y}_{1}=\varepsilon\left[\beta_{21} y_{2}(t-\tau)+\beta_{22} \dot{y}_{2}(t-\tau)\right] \\
& \ddot{y}_{2}+\omega_{2}^{2} y_{2}-\varepsilon\left(1-y_{2}^{2}-\alpha_{1} y_{1}^{2}\right) \dot{y}_{2}=\varepsilon\left[\beta_{11} y_{1}(t-\tau)+\beta_{12} \dot{y}_{1}(t-\tau)\right]
\end{aligned}
$$

where the dot denotes differentiation with respect to physical time $t, \varepsilon$ is a small positive parameter, $\tau$ is positive time delay. The origin of Eq. (1) can be found in Ref. [15] where bending-torsion vibrations of a thin beam under parametric excitation were discussed and a mathematical model without nonlinear dissipation and feedback control were represented. For simplicity, only one oscillator is parametrically excited in this paper. The present work is aimed at investigating the effects of feedback control and parametric excitation on response of such a nonlinearly coupled van der Pol system when 1:1 internal resonance and principal parametric resonance exist. The paper is arranged as follows. In the next section, the slow flow equations, which determine the principal parametric resonance response, are obtained with the aid of the averaging method. Their fixed points correspond to different types of solutions of the system considered. In Section 3, the stability of the trivial solution is discussed. In Sections 4 and 5, variation of the amplitude of periodic solutions of controlled system with excitation frequency and feedback parameters, based on Levenberg-Marquardt method, are given graphically. In section 6, simulations are presented to illustrate different kind of response when control parameters varied. The paper closes with a discussion, along with some conclusions in Section 7.

\section{Slow flow system}

For the case of principal parametric resonance and internal resonance, detuning parameters $\sigma_{1}$ and $\sigma_{2}$ are introduced such that

$$
\omega_{1}^{2}=\frac{\Omega^{2}}{4}+\varepsilon \sigma_{1}, \quad \omega_{2}^{2}=\omega_{1}^{2}+\varepsilon \sigma_{2}
$$

The Eq. (1) can be rewritten as

$$
\ddot{y}_{1}+\frac{\Omega^{2}}{4} y_{1}=\varepsilon F_{1}, \quad \ddot{y}_{2}+\frac{\Omega^{2}}{4} y_{2}=\varepsilon F_{2}
$$

where

$$
\begin{aligned}
& F_{1}=-\sigma_{1} y_{1}+2 f y_{2} \cos \Omega t+\left(1-y_{1}^{2}-\alpha_{2} y_{2}^{2}\right) \dot{y}_{1}+\left[\beta_{21} y_{2}(t-\tau)+\beta_{22} \dot{y}_{2}(t-\tau)\right] \\
& F_{2}=-\left(\sigma_{1}+\sigma_{2}\right) y_{2}+\left(1-y_{2}^{2}-\alpha_{1} y_{1}^{2}\right) \dot{y}_{2}+\left[\beta_{11} y_{1}(t-\tau)+\beta_{12} \dot{y}_{1}(t-\tau)\right]
\end{aligned}
$$

By the averaging method [16-18], the approximate solutions of Eq. (3) are supposed in the following form

$$
y_{i}=R_{i} \cos \left(\frac{\Omega}{2} t-\theta_{i}\right) \quad(i=1,2)
$$

where the amplitudes $R_{i}$ and the phases $\theta_{i}$ are time-dependent and governed by

$$
\begin{aligned}
\dot{R}_{i} & =-\varepsilon \frac{1}{4 \pi} \int_{0}^{\frac{4 \pi}{\Omega}} \sin \left(\frac{\Omega}{2} t-\theta_{i}\right) F_{i} d t \\
R_{i} \dot{\theta}_{i} & =\varepsilon \frac{1}{4 \pi} \int_{0}^{\frac{4 \pi}{\Omega}} \cos \left(\frac{\Omega}{2} t-\theta_{i}\right) F_{i} d t
\end{aligned}
$$


After integrating and truncating the Taylor expansions for those terms containing time delay [19,20], Eq. (6) is in the following form, namely, slow-flow equations

$$
\begin{aligned}
16 \Omega \dot{R}_{1}= & -R_{1}^{3} \Omega+4 R_{1} \Omega+4 \beta_{22} R_{2} \Omega \cos \left(\theta_{2}-\theta_{1}+\frac{1}{2} \Omega \tau\right)+R_{1} R_{2}^{2} \alpha_{2} \Omega \cos \left(2 \theta_{2}-2 \theta_{1}\right) \\
& +8 f R_{2} \sin \left(\theta_{1}+\theta_{2}\right)-2 R_{1} R_{2}^{2} \alpha_{2} \Omega-8 \beta_{21} R_{2} \sin \left(\theta_{2}-\theta_{1}+\frac{1}{2} \Omega \tau\right) \\
16 \Omega R_{1} \dot{\theta}_{1}= & -8 R_{1} \sigma_{1}+4 \beta_{22} R_{2} \Omega \sin \left(\theta_{2}-\theta_{1}+\frac{1}{2} \Omega \tau\right)+R_{1} R_{2}^{2} \alpha_{2} \Omega \sin \left(2 \theta_{2}-2 \theta_{1}\right) \\
& +8 f R_{2} \cos \left(\theta_{1}+\theta_{2}\right)+8 \beta_{21} R_{2} \cos \left(\theta_{2}-\theta_{1}+\frac{1}{2} \Omega \tau\right) \\
16 \Omega \dot{R}_{2}=- & R_{2}^{3} \Omega+4 \beta_{12} R_{1} \Omega \cos \left(\theta_{2}-\theta_{1}-\frac{1}{2} \Omega \tau\right)+4 R_{2} \Omega \\
+ & R_{2} R_{1}^{2} \alpha_{1} \Omega \cos \left(2 \theta_{2}-2 \theta_{1}\right)-2 R_{2} R_{1}^{2} \alpha_{1} \Omega+8 \beta_{11} R_{1} \sin \left(\theta_{2}-\theta_{1}-\frac{1}{2} \Omega \tau\right) \\
16 \Omega R_{2} \dot{\theta}_{2}= & -8 R_{2} \sigma_{1}-8 R_{2} \sigma_{2}-4 \beta_{12} R_{1} \Omega \sin \left(\theta_{2}-\theta_{1}-\frac{1}{2} \Omega \tau\right) \\
& -R_{2} R_{1}^{2} \alpha_{1} \Omega \sin \left(2 \theta_{2}-2 \theta_{1}\right)+8 \beta_{11} R_{1} \cos \left(\theta_{2}-\theta_{1}-\frac{1}{2} \Omega \tau\right)
\end{aligned}
$$

where the dot indicates differentiation with respect to the rescaled time $T=\varepsilon t$. Equation (7) is an autonomous dynamical system, the fixed points of which correspond to the periodic motions of the system described by Eq. (1). It is clearly seen that the addition of feedback control modifies the modulation equations. Consequently, the feedback control is possible to change the nonlinear dynamic characteristics of the system. There are four kinds of the fixed points of Eq. (7), namely, 1) $R_{1}=R_{2}=0$, 2) $\left.\left.R_{1}=0, R_{2} \neq 0,3\right) R_{1} \neq 0, R_{2}=0,4\right) R_{1} \neq 0, R_{2} \neq 0$. The first case corresponds to the trivial solution and the last three cases correspond to the periodic solutions of Eq. (1).

Letting $\tau=0$ in Eq. (7), the slow flow equations for the system under feedback control without time delay are obtained

$$
\begin{aligned}
16 \Omega \dot{R}_{1}= & -R_{1}^{3} \Omega+4 R_{1} \Omega+4 \beta_{22} R_{2} \Omega \cos \left(\theta_{2}-\theta_{1}\right)+8 f R_{2} \sin \left(\theta_{1}+\theta_{2}\right) \\
+ & R_{1} R_{2}^{2} \alpha_{2} \Omega \cos \left(2 \theta_{2}-2 \theta_{1}\right)-2 R_{1} R_{2}^{2} \alpha_{2} \Omega-8 \beta_{21} R_{2} \sin \left(\theta_{2}-\theta_{1}\right) \\
16 \Omega R_{1} \dot{\theta}_{1}= & -8 R_{1} \sigma_{1}+4 \beta_{22} R_{2} \Omega \sin \left(\theta_{2}-\theta_{1}\right)+R_{1} R_{2}^{2} \alpha_{2} \Omega \sin \left(2 \theta_{2}-2 \theta_{1}\right) \\
& +8 f R_{2} \cos \left(\theta_{1}+\theta_{2}\right)+8 \beta_{21} R_{2} \cos \left(\theta_{2}-\theta_{1}\right) \\
16 \Omega \dot{R}_{2}=- & R_{2}^{3} \Omega+4 \beta_{12} R_{1} \Omega \cos \left(\theta_{2}-\theta_{1}\right)+4 R_{2} \Omega \\
+ & R_{2} R_{1}^{2} \alpha_{1} \Omega \cos \left(2 \theta_{2}-2 \theta_{1}\right)-2 R_{2} R_{1}^{2} \alpha_{1} \Omega+8 \beta_{11} R_{1} \sin \left(\theta_{2}-\theta_{1}\right) \\
16 \Omega R_{2} \dot{\theta}_{2}= & -8 R_{2} \sigma_{1}-8 R_{2} \sigma_{2}-4 \beta_{12} R_{1} \Omega \sin \left(\theta_{2}-\theta_{1}\right) \\
& -R_{2} R_{1}^{2} \alpha_{1} \Omega \sin \left(2 \theta_{2}-2 \theta_{1}\right)+8 \beta_{11} R_{1} \cos \left(\theta_{2}-\theta_{1}\right)
\end{aligned}
$$

Similarly, the slow flow equations for the case without feedback control are obtained by letting $\beta_{11}=\beta_{12}=$ $\beta_{21}=\beta_{22}=0$ in Eq. (7):

$$
\begin{aligned}
& 16 \Omega \dot{R}_{1}=-R_{1}^{3} \Omega+4 R_{1} \Omega+R_{1} R_{2}^{2} \alpha_{2} \Omega \cos \left(2 \theta_{2}-2 \theta_{1}\right)+8 f R_{2} \sin \left(\theta_{1}+\theta_{2}\right)-2 R_{1} R_{2}^{2} \alpha_{2} \Omega \\
& 16 \Omega R_{1} \dot{\theta}_{1}=-8 R_{1} \sigma_{1}+R_{1} R_{2}^{2} \alpha_{2} \Omega \sin \left(2 \theta_{2}-2 \theta_{1}\right)+8 f R_{2} \cos \left(\theta_{1}+\theta_{2}\right) \\
& 16 \Omega \dot{R}_{2}=-R_{2}^{3} \Omega+4 R_{2} \Omega+R_{2} R_{1}^{2} \alpha_{1} \Omega \cos \left(2 \theta_{2}-2 \theta_{1}\right)-2 R_{2} R_{1}^{2} \alpha_{1} \Omega \\
& 16 \Omega R_{2} \dot{\theta}_{2}=-8 R_{2} \sigma_{1}-8 R_{2} \sigma_{2}-R_{2} R_{1}^{2} \alpha_{1} \Omega \sin \left(2 \theta_{2}-2 \theta_{1}\right)
\end{aligned}
$$

The nontrivial fixed point of (9) for the case of $\sigma_{1}=\sigma_{2}=0$ is $R_{1}=2, \quad R_{2}=0$ which corresponds to the periodic motion of the first oscillator. 


\section{Stability of the trivial solution}

The stability of the trivial solution of the system is associated with that of the fixed points of system (7), which is determined by the eigenvalues of the corresponding Jacobian matrix. To avoid the difficulty of solving these equations, we transform Eq. (7) into the form of the Cartesian coordinates [21] given by

$$
\begin{aligned}
& \dot{p}_{1}=\frac{1}{\Omega}\left(-\frac{1}{16} p_{1}^{3} \Omega-\frac{3}{16} p_{1} \alpha_{2} \Omega q_{2}^{2}-\frac{1}{16} p_{1} \alpha_{2} \Omega p_{2}^{2}-\frac{1}{16} p_{1} \Omega q_{1}^{2}+\frac{1}{4} p_{1} \Omega\right. \\
& -\frac{1}{2} \sigma_{1} q_{1}+\frac{1}{4} \beta_{22} \Omega \sin \left(\frac{1}{2} \Omega \tau\right) q_{2}+\frac{1}{8} p_{2} \alpha_{2} \Omega q_{2} q_{1}+\frac{1}{2} \beta_{21} \cos \left(\frac{1}{2} \Omega \tau\right) q_{2} \\
& \left.+\frac{1}{4} \beta_{22} \Omega p_{2} \cos \left(\frac{1}{2} \Omega \tau\right)-\frac{1}{2} \beta_{21} p_{2} \sin \left(\frac{1}{2} \Omega \tau\right)+\frac{1}{2} f q_{2}\right) \\
& \dot{q}_{1}=\frac{1}{\Omega}\left(-\frac{1}{16} \Omega q_{1} p_{1}^{2}+\frac{1}{2} p_{1} \sigma_{1}+\frac{1}{8} p_{1} p_{2} \alpha_{2} \Omega q_{2}-\frac{1}{2} \beta_{21} p_{2} \cos \left(\frac{1}{2} \Omega \tau\right)\right. \\
& +\frac{1}{4} \beta_{22} \Omega \cos \left(\frac{1}{2} \Omega \tau\right) q_{2}-\frac{3}{16} \alpha_{2} \Omega p_{2}^{2} q_{1}+\frac{1}{2} f p_{2}+\frac{1}{4} \Omega q_{1}-\frac{1}{4} \beta_{22} \Omega p_{2} \sin \left(\frac{1}{2} \Omega \tau\right) \\
& \left.-\frac{1}{16} \alpha_{2} \Omega q_{2}^{2} q_{1}-\frac{1}{16} \Omega q_{1}^{3}-\frac{1}{2} \beta_{21} \sin \left(\frac{1}{2} \Omega \tau\right) q_{2}\right) \\
& \dot{p}_{2}=\frac{1}{\Omega}\left(-\frac{1}{16} p_{2}^{3} \Omega-\frac{3}{16} p_{2} \alpha_{1} \Omega q_{1}^{2}+\frac{1}{4} p_{2} \Omega-\frac{1}{16} p_{2} \Omega q_{2}^{2}-\frac{1}{16} p_{2} \alpha_{1} \Omega p_{1}^{2}\right. \\
& -\frac{1}{2} \sigma_{2} q_{2}-\frac{1}{2} \sigma_{1} q_{2}+\frac{1}{8} p_{1} \alpha_{1} \Omega q_{1} q_{2}+\frac{1}{2} \beta_{11} \cos \left(\frac{1}{2} \Omega \tau\right) q_{1}+\frac{1}{4} \beta_{12} \Omega \cos \left(\frac{1}{2} \Omega \tau\right) p_{1} \\
& \left.+\frac{1}{4} \beta_{12} \Omega \sin \left(\frac{1}{2} \Omega \tau\right) q_{1}-\frac{1}{2} \beta_{11} \sin \left(\frac{1}{2} \Omega \tau\right) p_{1}\right) \\
& \dot{q}_{2}=\frac{1}{\Omega}\left(-\frac{1}{16} \Omega q_{2} p_{2}^{2}+\frac{1}{8} p_{1} p_{2} \alpha_{1} \Omega q_{1}+\frac{1}{2} p_{2} \sigma_{2}+\frac{1}{2} p_{2} \sigma_{1}-\frac{1}{16} \alpha_{1} \Omega q_{1}^{2} q_{2}\right. \\
& -\frac{1}{2} p_{1} \beta_{11} \cos \left(\frac{1}{2} \Omega \tau\right)-\frac{3}{16} \alpha_{1} \Omega p_{1}^{2} q_{2}-\frac{1}{4} p_{1} \beta_{12} \Omega \sin \left(\frac{1}{2} \Omega \tau\right) \\
& \left.+\frac{1}{4} \beta_{12} \Omega \cos \left(\frac{1}{2} \Omega \tau\right) q_{1}-\frac{1}{16} \Omega q_{2}^{3}+\frac{1}{4} \Omega q_{2}-\frac{1}{2} \beta_{11} \sin \left(\frac{1}{2} \Omega \tau\right) q_{1}\right)
\end{aligned}
$$

where $p_{1}=R_{1} \sin \left(\theta_{1}\right), q_{1}=R_{1} \cos \left(\theta_{1}\right), p_{2}=R_{2} \sin \left(\theta_{2}\right), q_{2}=R_{2} \cos \left(\theta_{2}\right)$.

The eigenvalues of the Jacobian matrix for the trivial solution are the roots of the following equation

$$
\lambda^{4}+J_{1} \lambda^{3}+J_{2} \lambda^{2}+J_{3} \lambda+J_{4}=0
$$

where $J_{1}=-\Omega$. According to Routh-Hurwitz criterion, it can be found that the trivial solution is always unstable.

\section{Periodic solutions of the controlled system without time delay}

Periodic solutions of the controlled system without time delay in feedback, which are determined by Eq. (8), is solved by Levenberg-Marquardt method [22] in this section. The relationships between parameters $\sigma_{1}, \sigma_{2}, \beta$ and the amplitudes $R_{1}, R_{2}$ are given in Figs 1 through 6 where solid and dashed lines indicate stable and unstable solutions respectively. For simplicity, parameters unchanged are chosen as $\Omega=2, \sigma_{1}=\sigma_{2}=0, \alpha_{1}=\alpha_{2}=1, \varepsilon=$ $0.1, f=5$ and $\beta_{21}=\beta_{22}=\beta_{11}=\beta_{12}=\beta=5$.

It can be seen that both of the two oscillators can be excited in periodic vibration with proper feedback. The amplitudes of the periodic vibration are related to the feedback gain, but they are independent of its sign. 


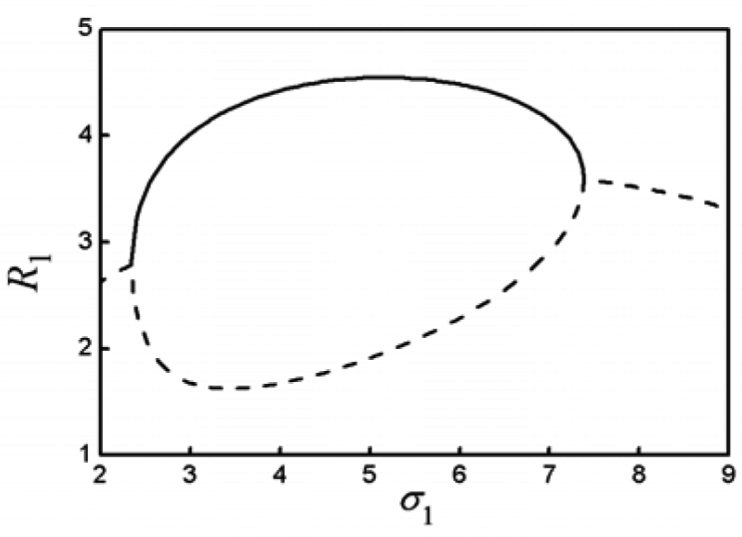

Fig. 1. Variation of $R_{1}$ with $\sigma_{1}$.

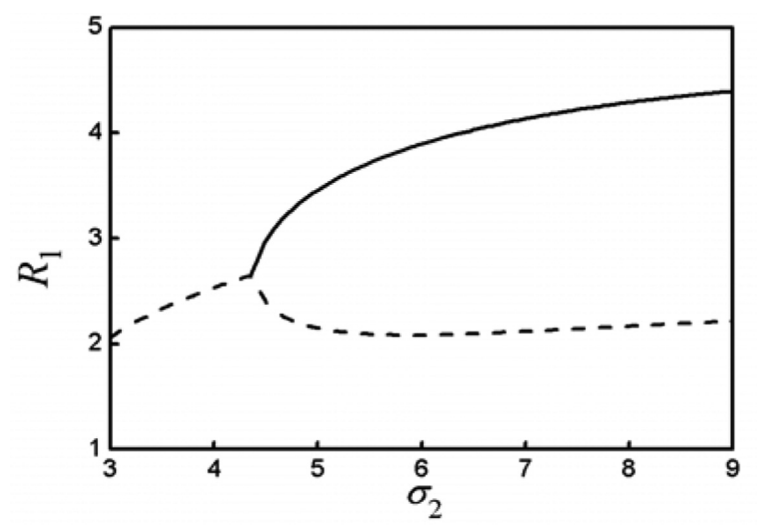

Fig. 3. Variation of $R_{1}$ with $\sigma_{2}$.

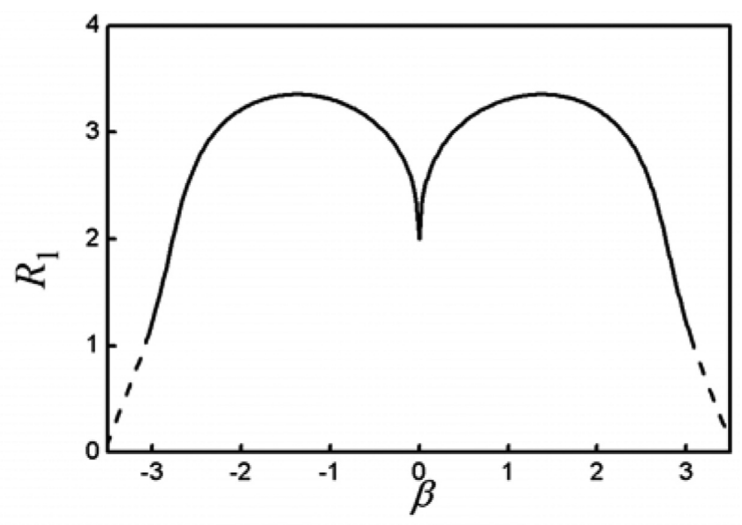

Fig. 5. Variation of $R_{1}$ with $\beta$.

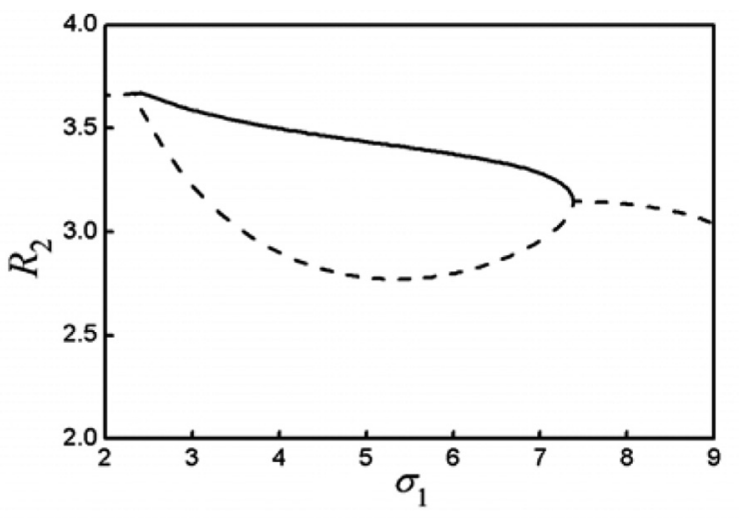

Fig. 2. Variation of $R_{2}$ with $\sigma_{1}$.

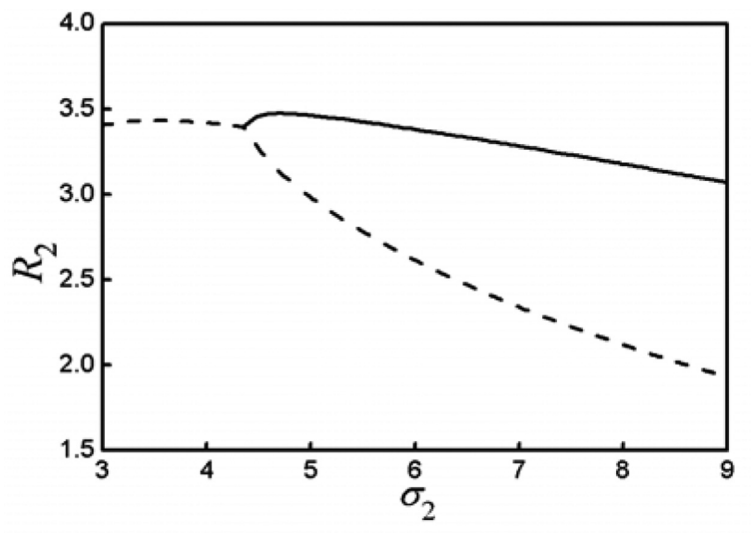

Fig. 4. Variation of $R_{2}$ with $\sigma_{2}$.

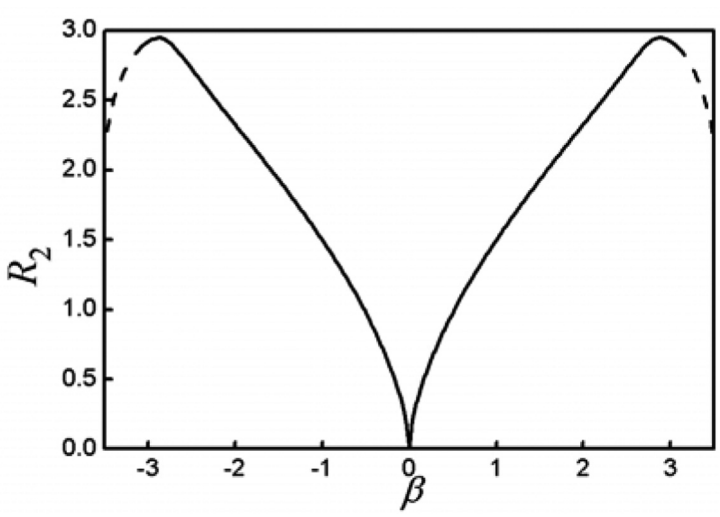

Fig. 6. Variation of $R_{2}$ with $\beta$.

\section{Periodic solutions of the controlled system with time delay}

In this section, Eqs (7) is solved by the same numerical method for the controlled systems with time delay in feedback. The relationships between control parameters and the amplitudes $R_{1}, R_{2}$ are given in Figs 7 through 10 .

It can be seen that the influence of time delay on the response of the system is periodic. Similar to the case without time delay in feedback, amplitude versus feedback gain are symmetrical about the origin. 


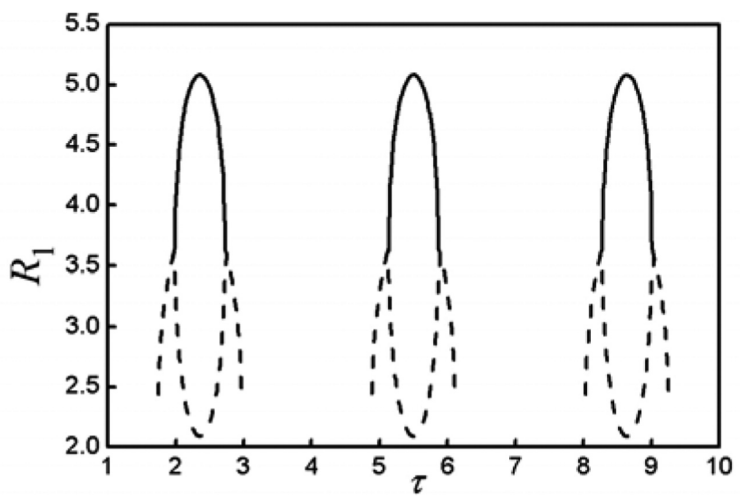

Fig. 7. Variation of $R_{1}$ with $\tau$.

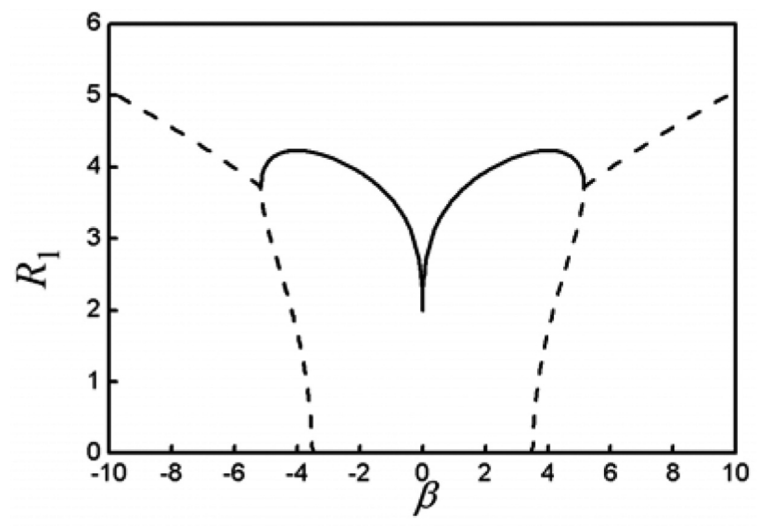

Fig. 9. Variation of $R_{1}$ with $\beta, \tau=2$.

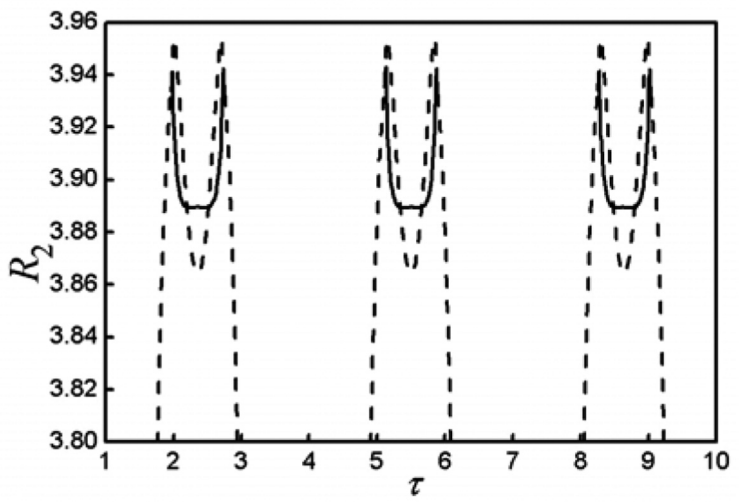

Fig. 8. Variation of $R_{2}$ with $\tau$.

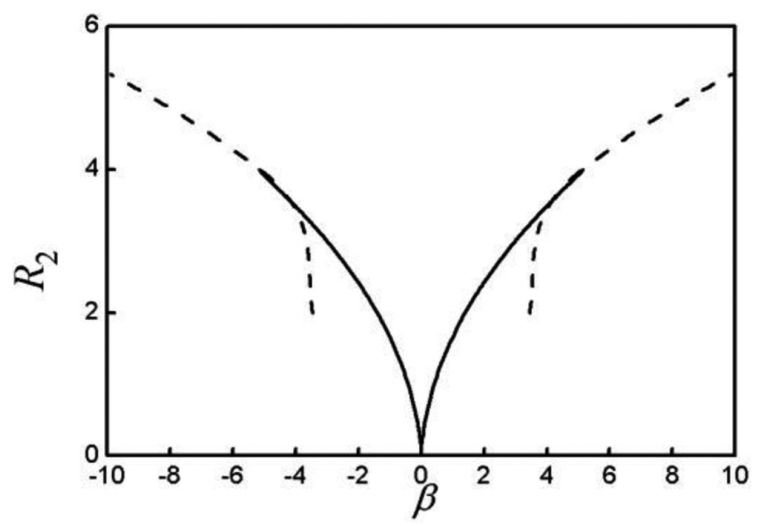

Fig. 10. Variation of $R_{2}$ with $\beta, \tau=2$.

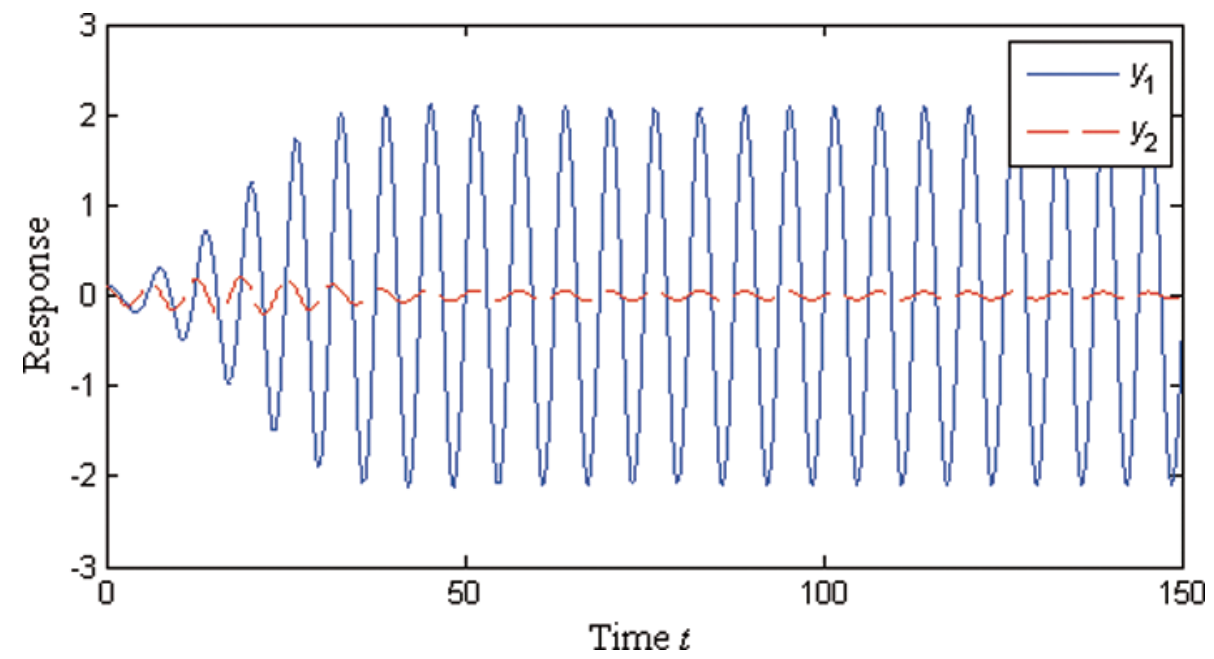

Fig. 11. Time history without feedback control. 


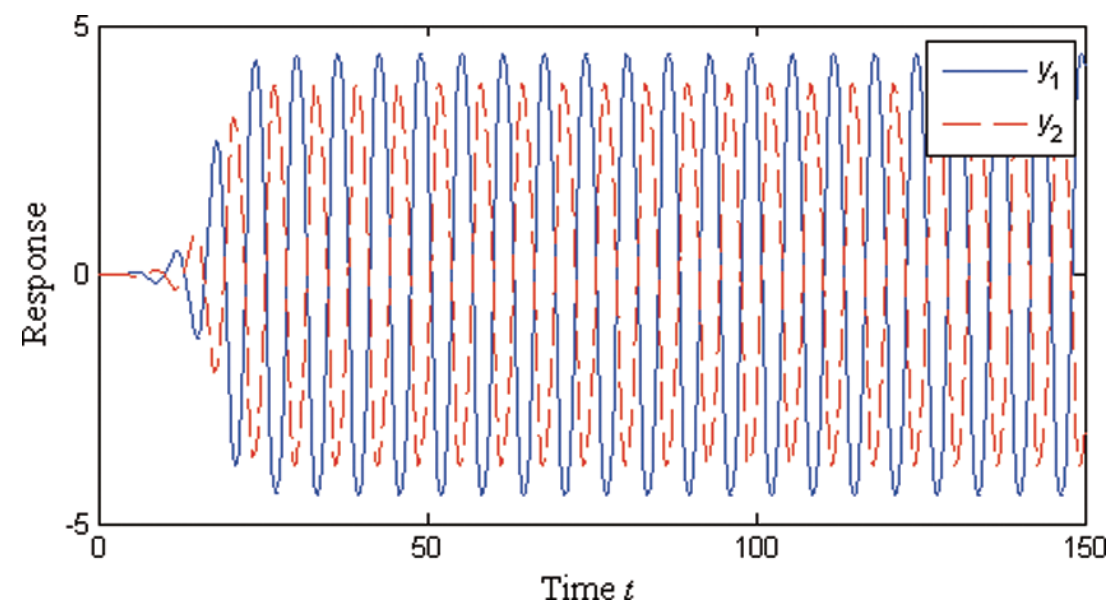

Fig. 12. Time history with feedback control, $\beta_{11}=\beta_{12}=\beta_{21}=\beta_{22}=5$.

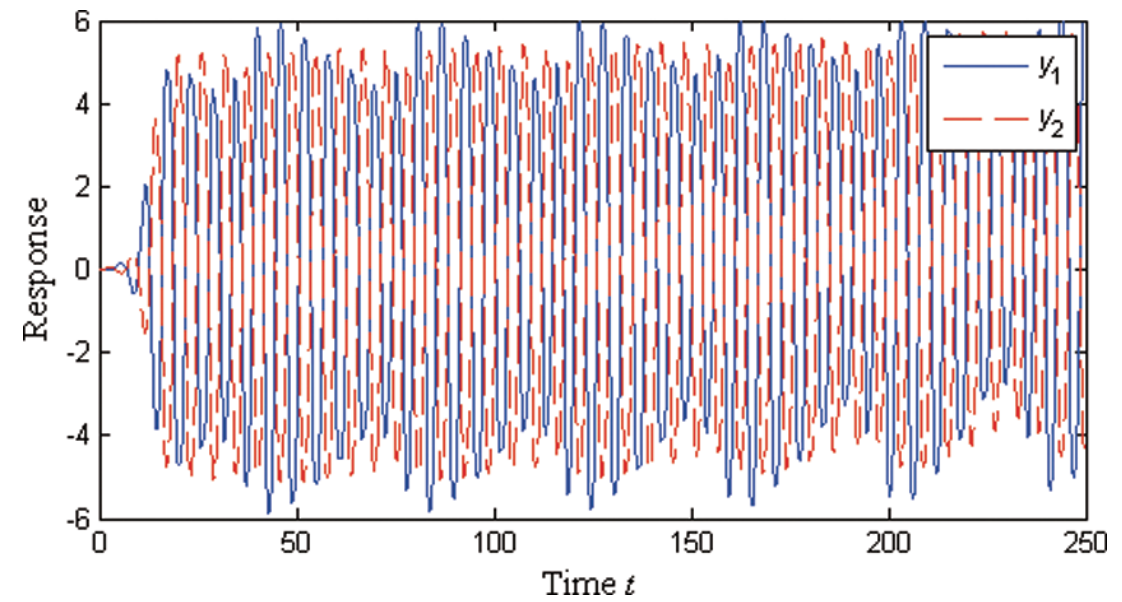

Fig. 13. Time history with feedback control with assumed parameters.

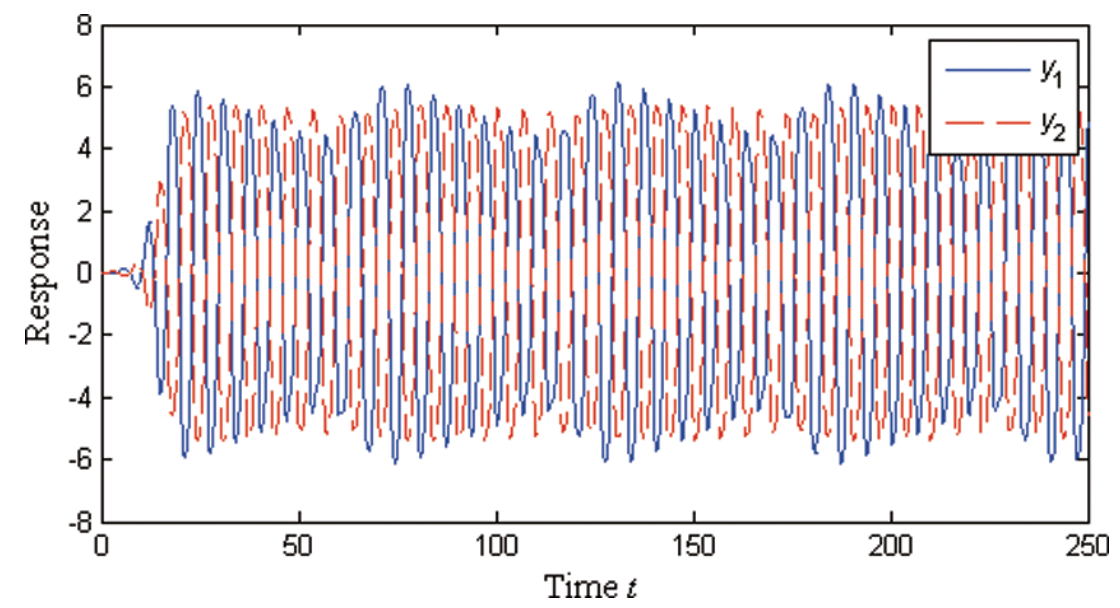

Fig. 14. Time history with feedback control, $\tau=2.5$. 


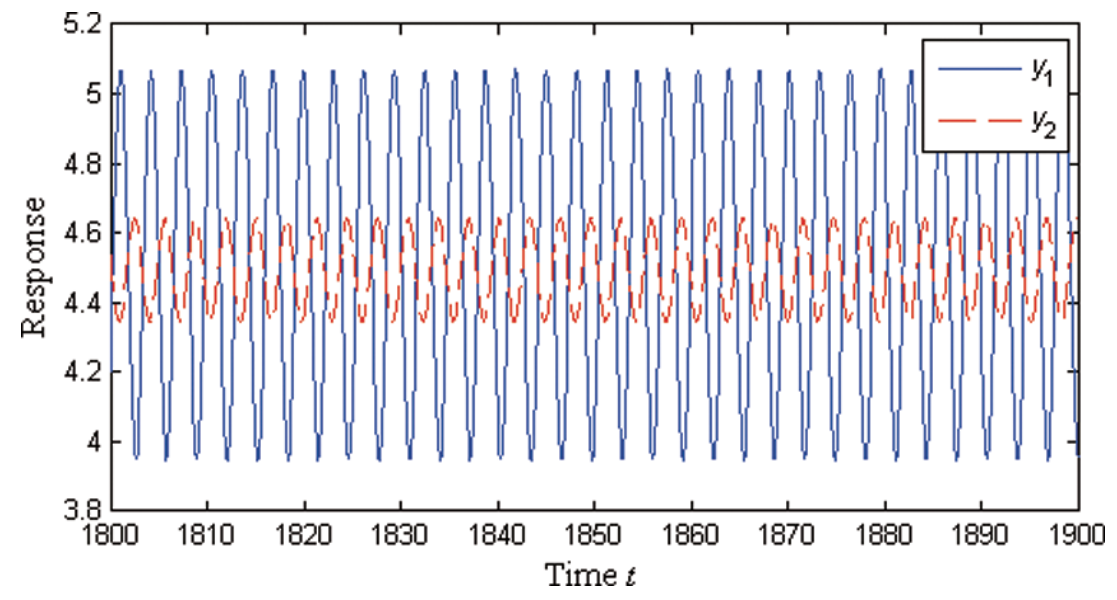

Fig. 15. Time history with feedback control, $\tau=1$.

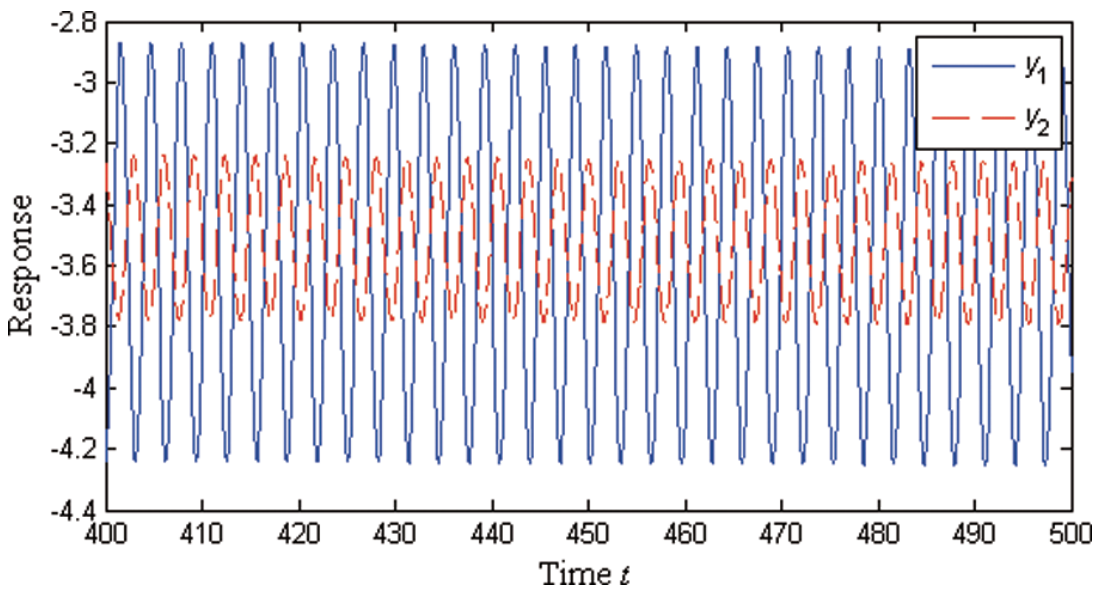

Fig. 16. Time history with feedback control, $\tau=4$.

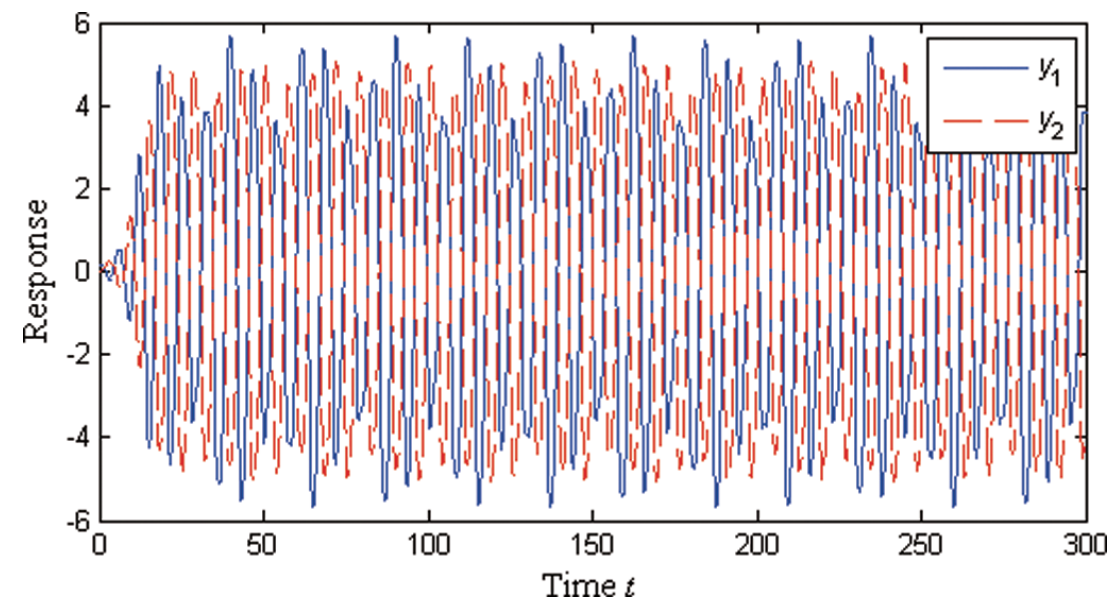

Fig. 17. Time history with position feedback only, $\left(y_{1}, y_{2}, \dot{y}_{1}, \dot{y}_{2}\right)=(0.1,0.1,0,0)$. 
(a) Time history

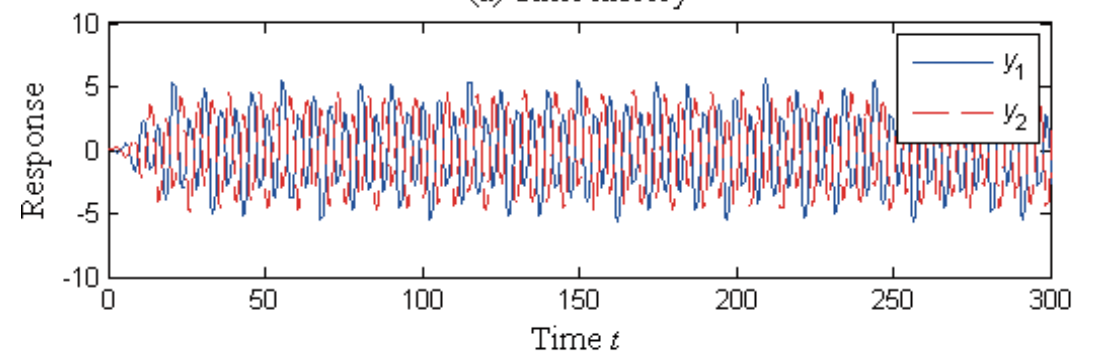

(b) Phase trajectory in $y_{1}-\mathrm{d} y_{1} / \mathrm{d} t$ plane (c) Phase trajectory in $y_{2}-\mathrm{d} y_{2} / \mathrm{d} t$ plane
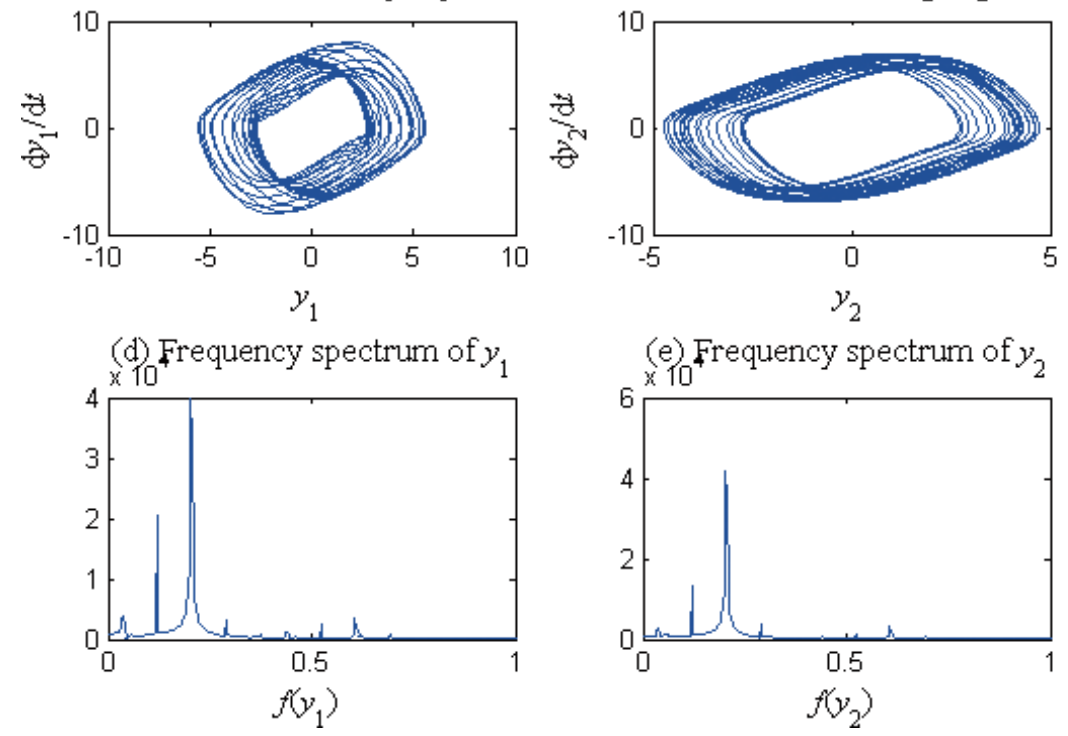

(e) Frequency spectrum of $y_{2}$

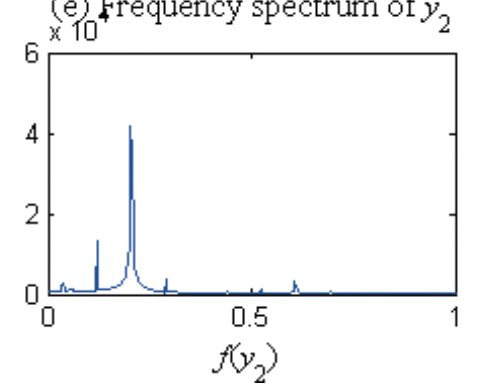

Fig. 18. Case with velocity feedback only, $\left(y_{1}, y_{2}, \dot{y}_{1}, \dot{y}_{2}\right)=(0.1,0.1,0,0)$.

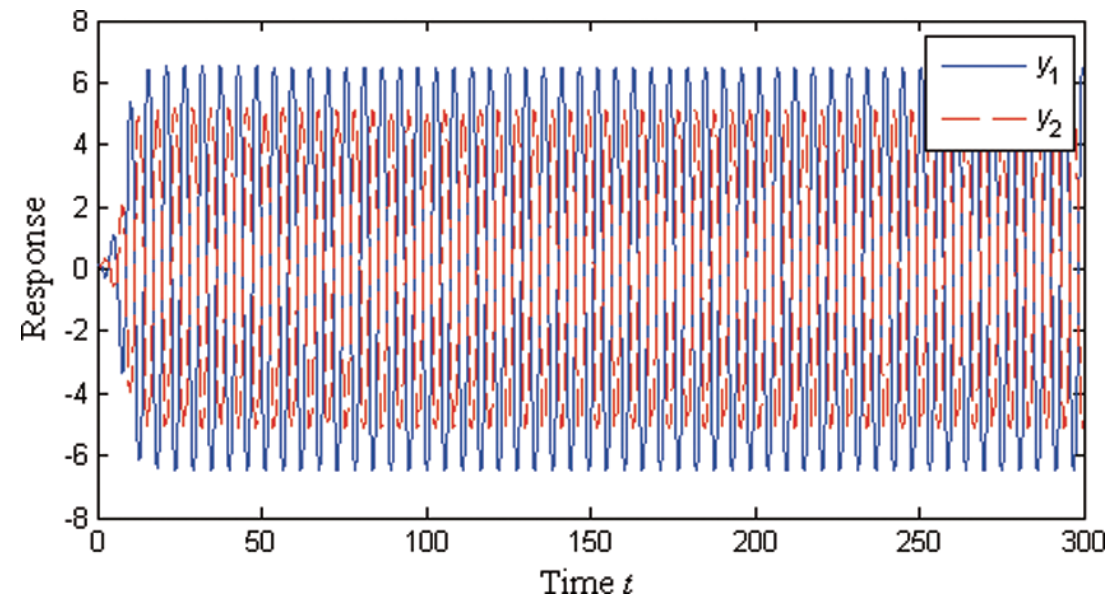

Fig. 19. Time history, $\Omega=2.3,\left(y_{1}, y_{2}, \dot{y}_{1}, \dot{y}_{2}\right)=(0.1,0.1,0,0), f=10$. 


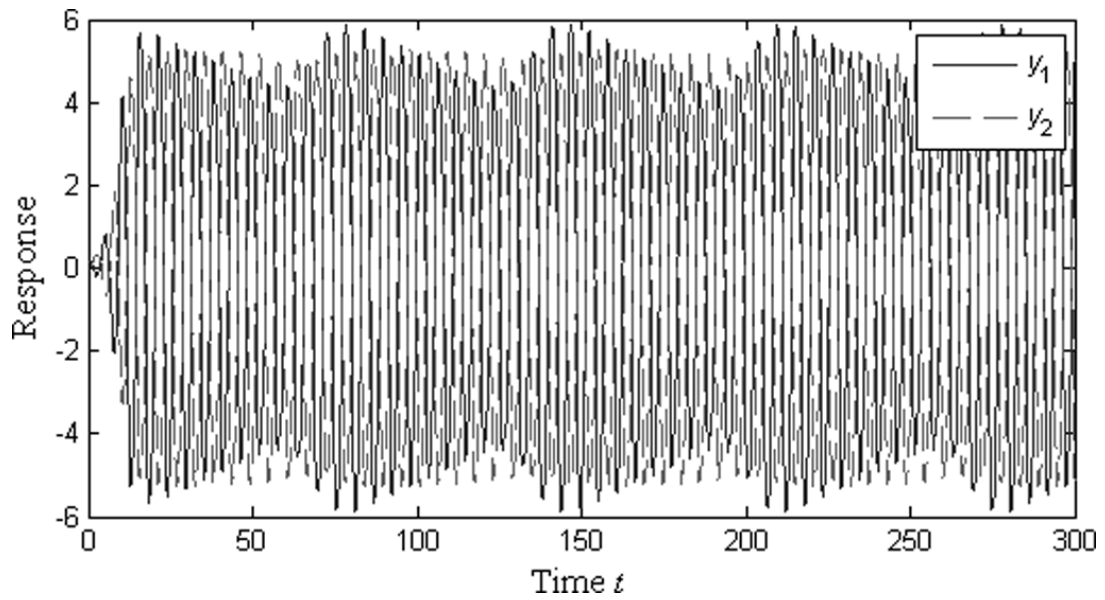

Fig. 20. Time history, $\Omega=2.3\left(y_{1}, y_{2}, \dot{y}_{1}, \dot{y}_{2}\right)=(0.1,0.1,0,0)$.

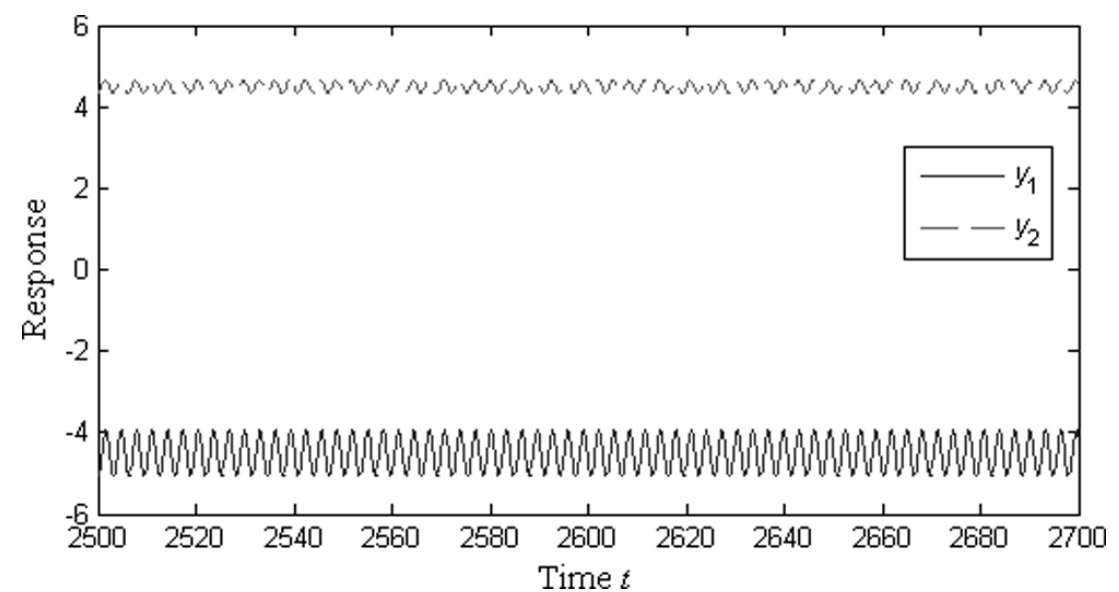

Fig. 21. Time history with feedback control, $\tau=1, \beta_{11}=\beta_{12}=\beta_{21}=\beta_{22}=-10,\left(y_{1}, y_{2}, \dot{y}_{1}, \dot{y}_{2}\right)=(0.5,0.5,0,0)$.

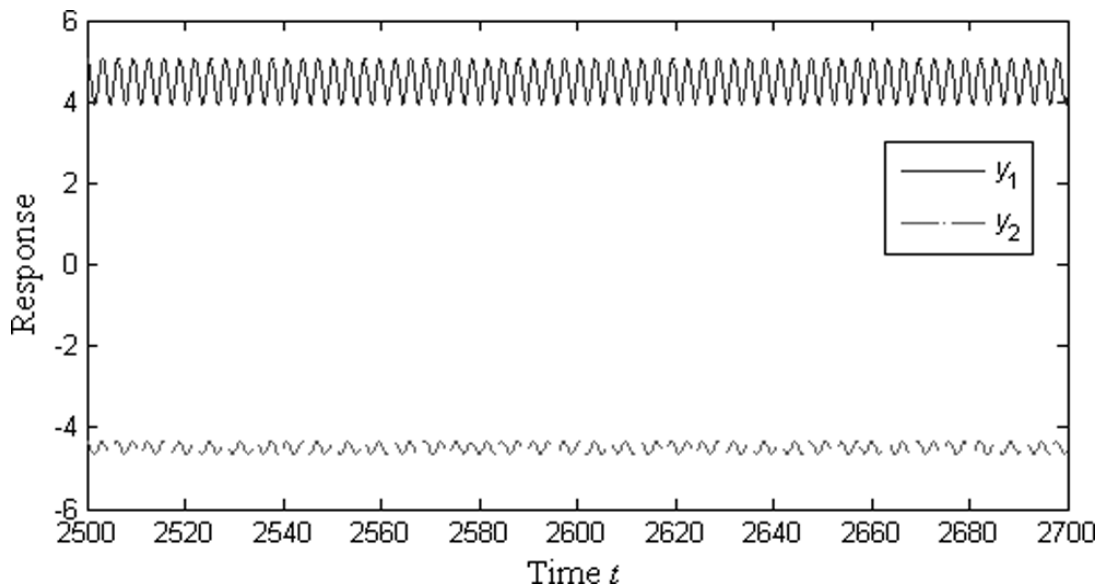

Fig. 22. Time history with feedback control, $\tau=1, \beta_{11}=\beta_{12}=\beta_{21}=\beta_{22}=-10,\left(y_{1}, y_{2}, \dot{y}_{1}, \dot{y}_{2}\right)=(0.5,0.5,0,0)$. 


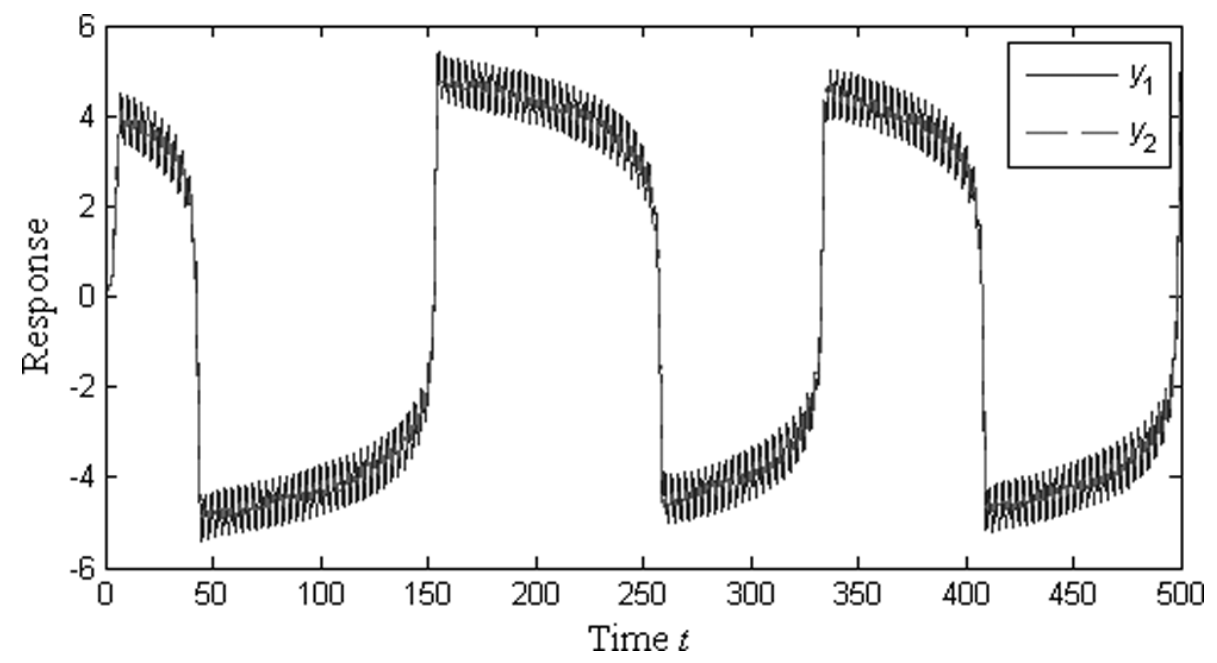

Fig. 23. Time history with feedback control, $\tau=0,\left(y_{1}, y_{2}, \dot{y}_{1}, \dot{y}_{2}\right)=(0.01,0.01,0,0)$.

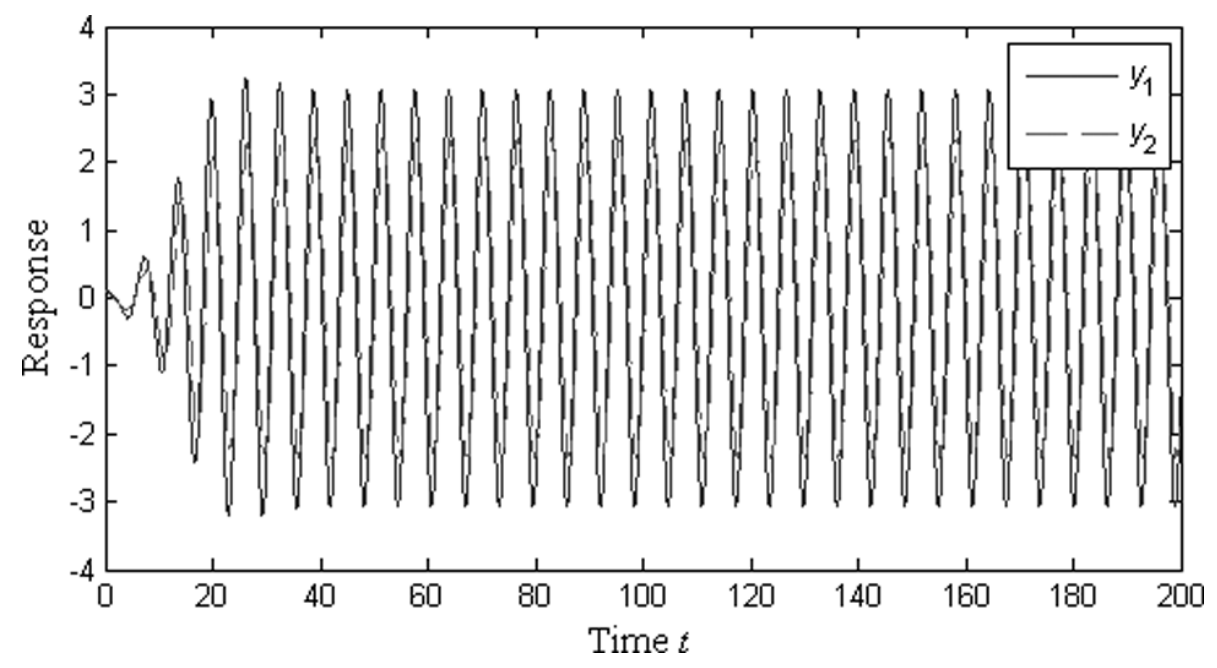

Fig. 24. Time history with feedback control, $\tau=0, \beta_{11}=\beta_{12}=\beta_{21}=\beta_{22}=2$.

\section{Simulations}

In this section, numerical simulations are given to show the effect of control and excitation parameters based on MATLAB program. The default of feedback gains is $\beta_{21}=\beta_{22}=\beta_{11}=\beta_{12}=10$. Otherwise, the results are denoted by special values of parameters. Initial condition for numerical integral is $\left(y_{1}(0), y_{2}(0), \dot{y}_{1}(0), \dot{y}_{2}(0)\right)=$ $(0.01,0.01,0,0)$.

To compare the results of cases with and without feedback control, the time history for the latter is first given in Fig. 11. It is shown that the indirectly excited oscillator keeps rest and the directly excited one has a steady state response with amplitude 2 and angular frequency $\Omega / 2$, which is consistent with the analysis. This conclusion is not dependent on initials. The Fig. 12, in which both of the two oscillators have steady state response, is given with different feedback gain $\beta_{11}=\beta_{12}=\beta_{21}=\beta_{22}=5$. It is shown that the trivial solution is unstable and the feedback gain can greatly affect the dynamical behavior of the system. Figures 13 through 16 are given for different time delay in feedback respectively. It is shown that time delay in feedback also have great influence on the dynamical behavior of the system. Especially, it is found that the equilibrium position can shift as the result of time delay in feedback as shown in Figs 15 and 16. Figures 17 and 18 are given for the cases of position and velocity feedback 
respectively. It is shown that they can play same role in control, which can be verified by the slow flow Eqs (7) and (8). By the phase trajectories and frequency spectrums in Fig. 18, it is shown that the response is quasi-periodic motion for assumed values of parameters when only velocity feedback is available. Figures 19 and 20, corresponding to periodic and quasi-periodic motion respectively, are given for different excitation amplitude with frequency $\Omega=$ 2.3. It is shown that excitation amplitude also has great influence on the dynamical behavior of the system. Another important phenomenon is that the steady state responses of the system with feedback may dependent on the initial conditions for some parameters as shown in Figs 21 and 22. The equilibrium positions of the two oscillators shift to opposite directions, which is different from the case in Figs 15 and 16. Figures 23 and 24, corresponding to relaxation oscillation [16] and periodic motion of in-phase mode respectively, are given for the case without time delay in feedback control.

\section{Discussion and conclusion}

The averaging method and numerical simulations are employed in this paper to study the principal parametric resonance of two van der Pol oscillators on the assumption that only one of the two oscillators is parametrically excited and coupled state feedback control is linear. Time delay in feedback and 1:1 internal resonance are considered. For the uncontrolled system, nontrivial solution exists only for one oscillator. Similar to the single van der Pol oscillator, the amplitude of the limit circle is 2 . It is found that the trivial solution of the controlled system is always unstable even if feedback control is applied. Based on Levenberg-Marquardt method, the variation of amplitude of periodic solutions with feedback gain and time delay is given graphically. It can be seen that both of the two oscillators can be excited in periodic vibration with proper feedback. However, the amplitudes of the periodic vibration are independent of the sign of feedback gain. In addition, the influence of time delay on the response of the system is periodic. In terms of numerical simulations, it is shown that the two oscillators may also have quasi-periodic motions, periodic motions about a new equilibrium position and other complex phenomenon such as relaxation oscillation when feedback control is applied.

\section{Acknowledgments}

This research is supported by the National Natural Science Foundation of China under Grant No.10872063. The authors would like to thank the reviewers for their valuable suggestions.

\section{References}

[1] L.A. Low, P.G. Reinhall and D.W. Storti, An investigation of coupled van der Pol oscillators, Journal of vibration and acoustics 125 (2003), $162-169$.

[2] J. Guckenheimer and P. Holmes, Nonlinear Oscillation and Bifurcation of Vector Field, Springer-Verlag, New York, 1993.

[3] W. Zhang and Y. Chen, Development of modern theory of nonlinear dynamical systems with parametric excitation, Advances in Mechanics 28 (1998), 1-16 (in Chinese).

[4] A. Maccari, Modulated motion and infinite-period bifurcation for two nonlinearly coupled and parametrically excited van der Pol oscillators, International Journal of Nonlinear Mechanics 36 (2001), 335-347.

[5] A.Maccari, Parametric excitation for two internally resonant van der Pol oscillators, Nonlinear Dynamics 27 (2002), 367-383.

[6] T. Bakri, R. Nabergoj, A. Tondl and F. Verhulst, Parametric excitation in non-linear dynamics, International Journal of Nonlinear Mechanics 39 (2004), 311-329.

[7] S. Fatimah and M. Ruijgrok, Bifurcations in an auto-parametric system in 1:1 internal resonance with parametric excitation, International Journal of Nonlinear Mechanics 37 (2002), 297-308.

[8] W. Han, D. Jin and H. Hu, Dynamics and stability of a nonlinear controlled system subject to parametric excitation, Acta Mechanica Sinica 35 (2003), 303-308 (in Chinese).

[9] M. Ye, J. Lv, Q. Ding and W. Zhang, The bifurcation analysis on the laminated composite plate with 1:1parametric resonance, Acta Mechanica Sinica 36 (2004), 64-71 (in Chinese).

[10] W. Zhang, H. Wen and M. Yao, Periodic and chaotic oscillation of a parametrically excited viscoelastic moving belt with 1:3 internal resonance, Acta Mechanica Sinica 36 (2004), 443-454 (in Chinese). 
[11] Q. Bi, Dynamical analysis of coupled parametrically excited van der Pol oscillators, International Journal of Nonlinear Mechanics 39 (2002), 33-54.

[12] J. Xu and L. Pei, Advances in dynamics for delayed systems, Advances in Mechanics 36 (2006), 17-30 (in Chinese).

[13] A.F. EL-Bassiouny, Fundamental and subharmonic resonances of harmonically oscillation with time delay state Feedback, Shock and Vibration 13 (2006), 65-83.

[14] L. Jun, R. Shen and H. Hua, Cubic velocity feedback control of high-amplitude vibration of a nonlinear plant to a primary resonance excitation, Shock and Vibration 14 (2007), 1-14.

[15] J. Dugundji and V. Mukhopadhyay, Lateral bending-torsion vibrations of a thin beam under parametric excitation, Journal of Applied Mechanics 40 (1973), 693-698.

[16] A.H. Nayfeh and D.T. Mook, Non-linear Oscillations, Wiley, New York, 1979.

[17] A.H. Nayfeh, Perturbation Techniques, Wiley, New York, 1981.

[18] A.H. Nayfeh and B. Balachandran, Applied Nonlinear Dynamics, Wiley, New York, 1995.

[19] S. Wirkus and R. Rand, The dynamics of two van der Pol oscillators with delay coupling, Nonlinear Dynamics 30 (2002), $205-221$.

[20] X.Y. Li, Y.S. Chen, Z.Q. Wu and T. Song, Response of parametrically excited Duffing-van der Pol oscillator with delayed feedback, Applied Mathematics and Mechanics 27 (2006), 1585-1595.

[21] J.C. Ji and A.Y.T. Leung, Bifurcation control of a parametrically excited Duffing system, Nonlinear Dynamics 27 (2002), $411-417$.

[22] J. More, Numerical Analysis, Springer, New York, 1978. 

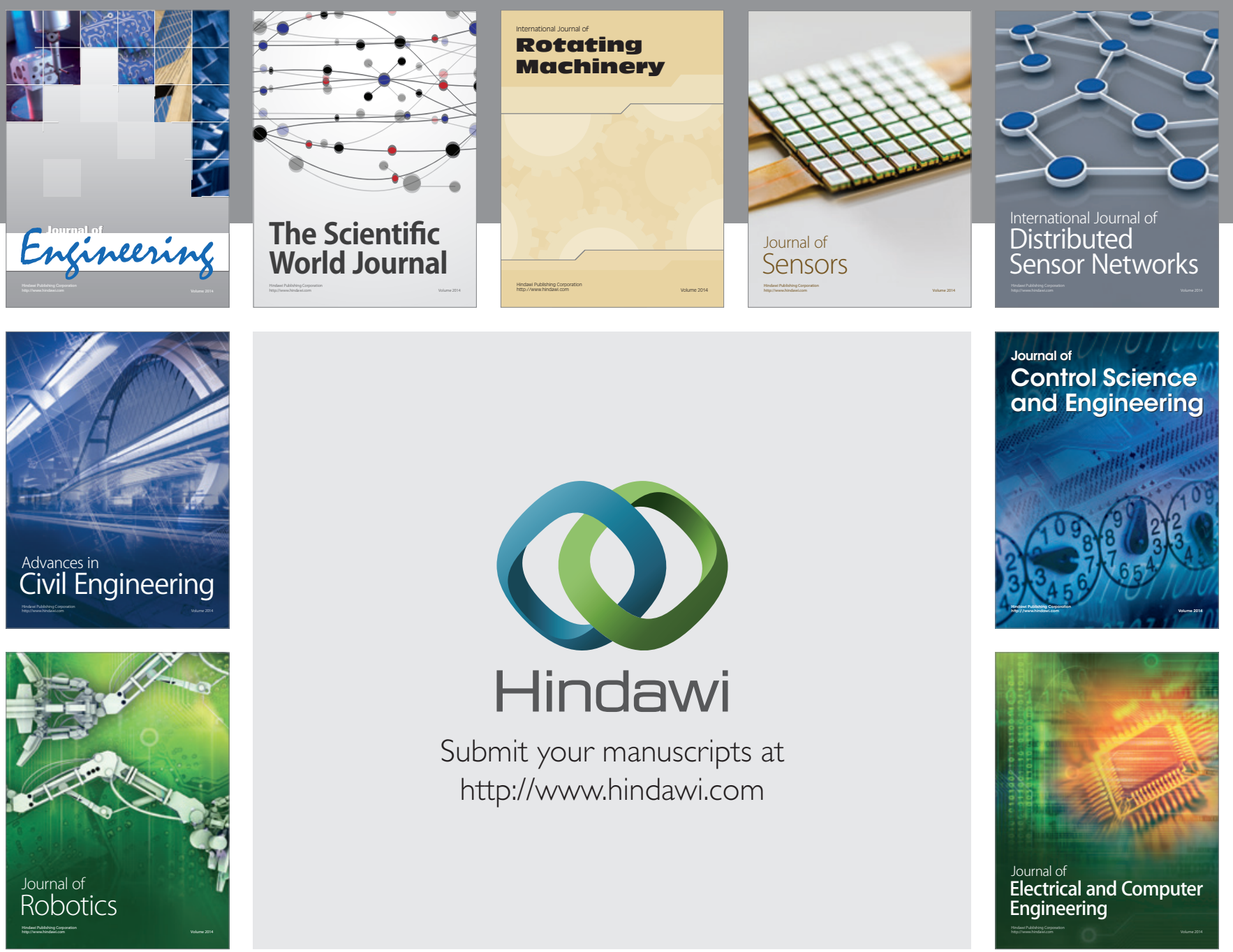

Submit your manuscripts at

http://www.hindawi.com
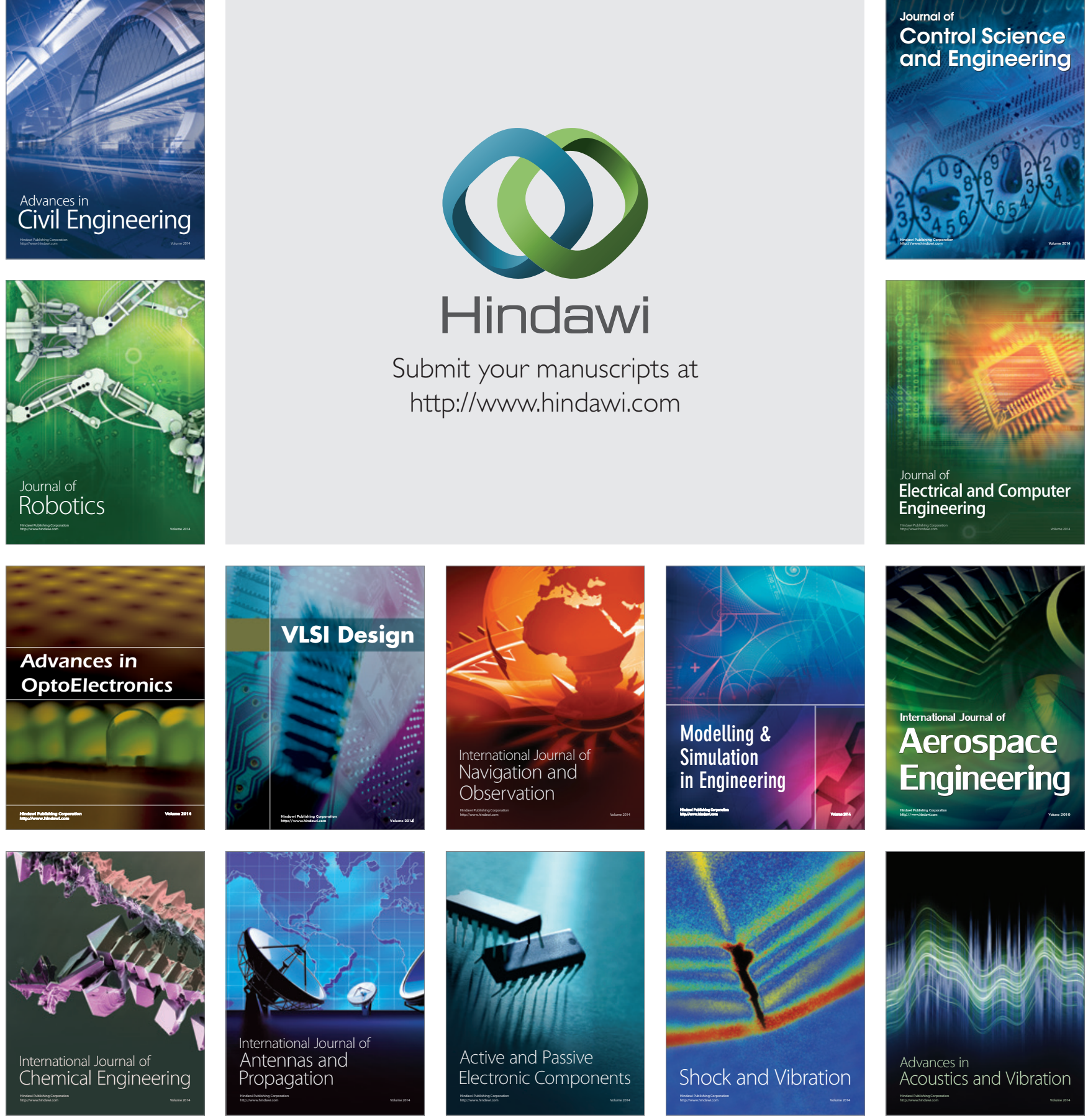